\title{
Numerical Analysis and Diagnosis of the Hydrodynamic Effects Produced by Hurricane Gordon along the Coast of Spain
}

\author{
Gabriel Diaz-Hernandez, Fernando J. Mendez, And Roberto Mínguez \\ Environmental Hydraulics Institute, Santander, Cantabria, Spain
}

(Manuscript received 7 November 2013, in final form 24 February 2014)

\begin{abstract}
This paper presents a detailed hindcast for the generation and propagation of sea state variables — significant wave height $H_{s}$, peak period $T_{p}$, mean direction $\theta$, and spectral shape $\gamma-\sigma$-associated with cyclonic events to numerically diagnose their possible hydrodynamic effects over the northeastern Atlantic. An example of such cyclonic events is Hurricane Gordon, which occurred during the second half of August 2012. Extreme hurricane-strength winds produced new and atypically low-frequency (about $14 \mathrm{~s}$ ) packs of energy. The preexistent wave spectrum suddenly experienced an addition of low-frequency energy along the coast of Cádiz, Spain. This study presents the results of a comprehensive analysis developed to reconstruct the events produced by Hurricane Gordon (2012) along the coast of Cádiz. The analysis features the use of (i) parametric models for the characterization of hurricane winds and pressure fields, (ii) implementation of the Simulating Waves Nearshore (SWAN) model for the generation and propagation of waves in the northeast Atlantic Ocean, and (iii) its coupling with the MOPLA — taken from the Spanish acronym for wave propagation model, current, and morphodynamic evolution of beaches-model for the evaluation of longshore currents. The numerical wave characterization, generation, and propagation were validated with instrumental data from deep-water and coastal buoys.
\end{abstract}

\section{Introduction}

Hurricane Gordon (2012) belongs to the family of North Atlantic hurricanes that move from west to east and beyond $26^{\circ} \mathrm{W}$ longitude. It started as a tropical storm, reaching hurricane category 3 on the Saffir-Simpson scale with sustained wind fields above $200 \mathrm{~km} \mathrm{~h}^{-1}$. This family of hurricanes is characterized by low probability of occurrence, with one to three cyclonic events per decade based on historical records from 1850 to 2012 from the North Atlantic hurricane database (HURDAT; Jarvinen et al. 1984; Landsea et al. 2004, 2008). Some examples of this type of event are Carrie (1957, category 1), Hannah (1959, category 2), Debbie (1961, category 1), Fran (1973, category 1), Emmy (1976, category 1), Floyd (1993, category 1), Gordon (2006, category 1), and Vince (2005, category 1), among others.

According to the official report of Hurricane Gordon (Avila 2012), this storm reached category 2 status and

Corresponding author address: Gabriel Diaz-Hernandez, Environmental Hydraulics Institute, Universidad de Cantabria, c/ Isabel Torres 15, Parque Cientfico y Tecnológico de Cantabria, Santander 39011, Cantabria, Spain.

E-mail: diazg@unican.es weakened to a category 1 as it made landfall at the city of Santa Maria in the Azores Islands, Portugal (see Fig. 1). It produced flooding in several coastal areas of the Azores, downed trees, and caused power outages and landslides, without, however, significant material losses or deaths.

Presumably, Hurricane Gordon was the direct cause of a sudden and unusual pattern of behavior in the coastal hydrodynamics along the coast of Cádiz. Between 21 and 22 August 2012, low-severity swell $\left(H_{s} \approx\right.$ $1 \mathrm{~m})$ and high peak periods $\left(T_{p} \approx 14 \mathrm{~s}\right.$ ) reached the coast of Cádiz. This sea state induced high-energy rip currents throughout this area, whose strength compromised the safety of bathers on the coast forcing emergency actions to be taken by the Cádiz Rescue Department.

This atypical behavior in coastal currents is attributed to an added amount of external energy, produced by the passage of the hurricane along the eastern Atlantic Ocean. Radical changes in the incoming wave spectral energy were observed, before and after the cyclone event. The aim of this article is to present the methodology used to generate, propagate, and interact with the waves resulting from Hurricane Gordon's approach toward the surf zone and is based on a coupled system of numerical models. 


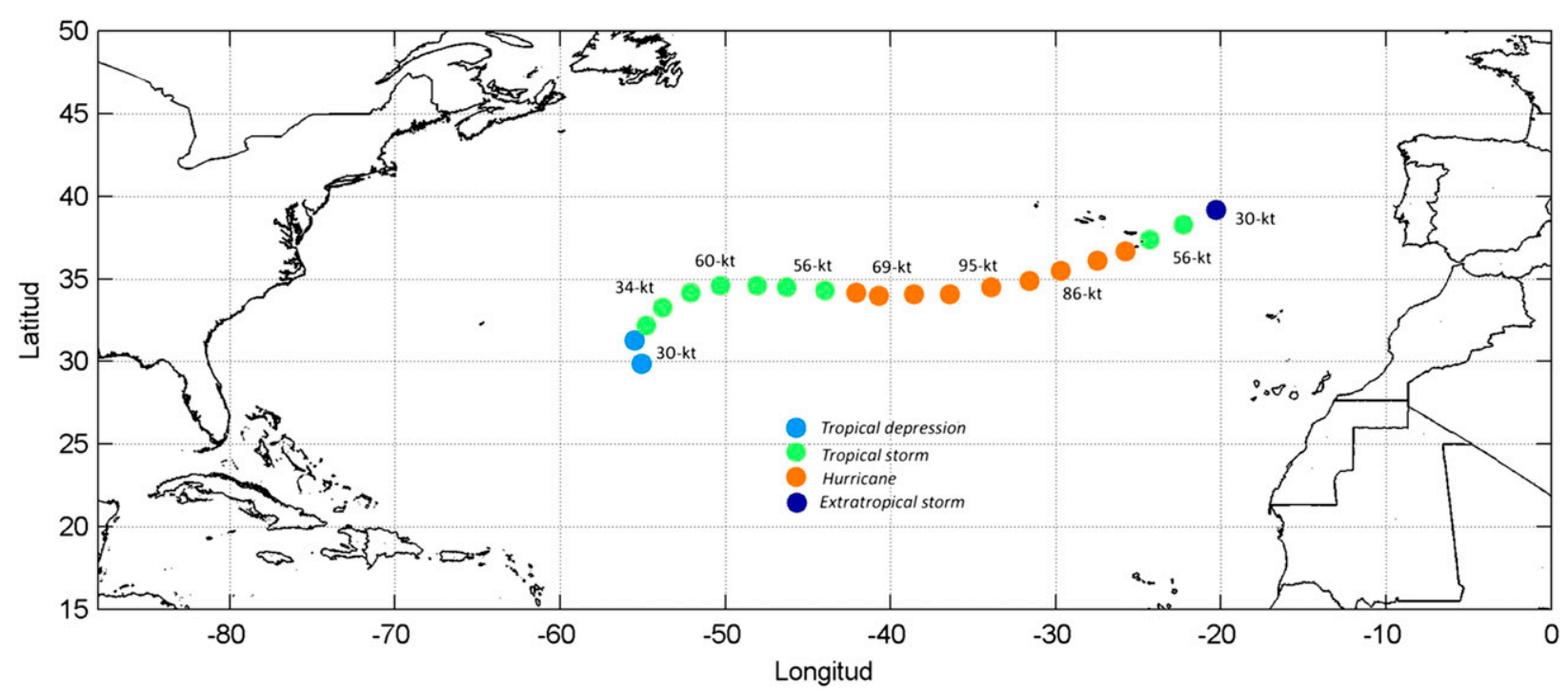

FIG. 1. Best track for Hurricane Gordon, August 2012.

This manuscript is organized as follows. Section 2 describes the characteristics of the synoptic wind and instrumental data, which have been used as forcing for the Simulating Waves Nearshore (SWAN) numerical model (Zijlema 2010) and validation, respectively, paying special attention to the adaptation of high-resolution wind fields associated with hurricanes. Section 3 describes the use and implementation of both the SWAN and MOPLA (taken from the Spanish acronym for wave propagation model, current, and morphodynamics evolution of beaches) models (González et al. 2007). Section 4 presents the numerical wave propagation associated with Hurricane Gordon as it made its way toward the coast of Cádiz. In section 5, MOPLA's wave height and breaking current results following the propagation waves using SWAN to the coastal area are presented and discussed. Finally, in section 6 some relevant conclusions are drawn.

\section{Input data and models}

While Hurricane Gordon moved across the northeast Atlantic, several wave and wind measurements were acquired at different coastal and ocean locations. This information is crucial to our understanding and reproducing the generation, evolution, and performance of these variables on the days before, during, and after the storm. This section presents the general characteristics of instrumental data and numerical output that describe the wave and wind fields, respectively. This dataset is used both as forcing for the SWAN numerical model and for its validation. In addition, this section presents the preprocessing of wind fields, especially as related to the hurricane, for an appropriate implementation within the SWAN model.

\section{a. Bathymetry}

The bathymetry implemented in this study results from the integration of high-resolution bathymetry charts 443, 444, and 445 of the Spanish Navy Hydrographic Service, as well as General Bathymetric Chart of the Oceans (GEBCO) data, which cover the spatial domain $25^{\circ}-43^{\circ} \mathrm{N}$ and $65^{\circ}-5^{\circ} \mathrm{W}$. This domain comprises a coastline of about $100 \mathrm{~km}$ (see Fig. 2), including the entire coast of Cádiz from the city of Sanlucar de Barrameda to the north to the city of Tarifa to the south.

\section{b. Measurements data}

In Spain, the Ports Agency manages different oceanographic measurement sensors located both in deep and shallow waters. The first measurement campaign started in 1981 within the REMRO (from the Spanish acronym for wave buoy network) program, which included 21 scalar buoys moored in deep waters. In late 1987, a new campaign started, incorporating directional and weather information and constituting a real-time measurement network that combined REDCOS (from the Spanish acronym for coastal buoy network) and REDMAR (from the Spanish acronym for tide gauge network) data (Ruiz et al. 1995). These buoys were installed as a permanent measurement network and record real-time series of meteorological-ocean parameters, providing the longest instrumental database for Spain. Buoy records include time series of wave elevations above mean sea level, wave height, and period and directional components, such as lift, roll, pitch, and yaw. Currently, this network has 25 


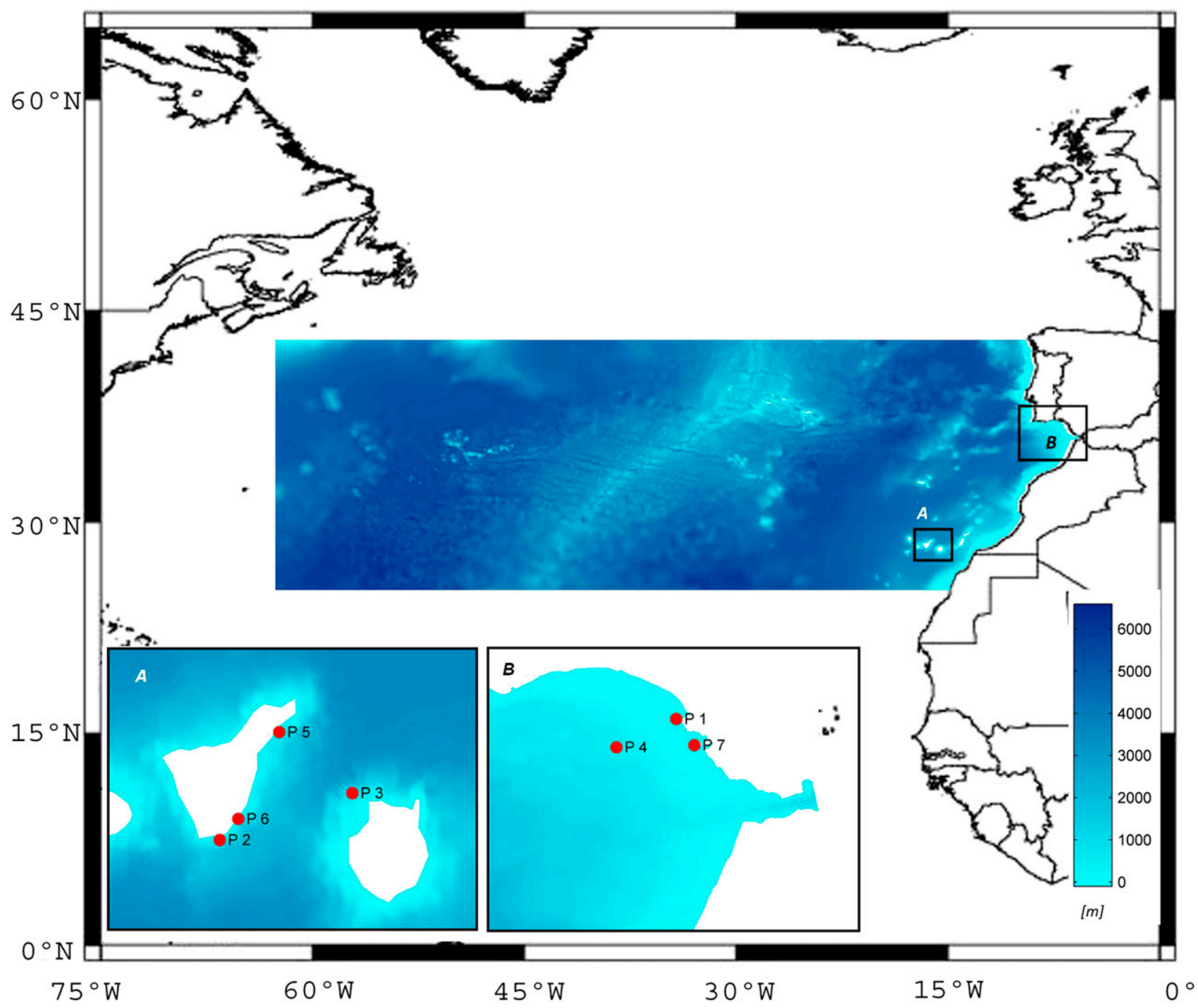

FIG. 2. Bathymetry provided by GEBCO for the $25^{\circ}-43^{\circ} \mathrm{N}$ and $65^{\circ}-5^{\circ} \mathrm{W}$ area. Insets (a),(b) show high-resolution bathymetry and locations of the buoys used in this study (red points).

stations, 14 of which are Triaxys directional buoys and 11 of which are scalar Waverider buoys.

In this study, these real-time wave data are used both as forcing and validation for wave propagation models. Table 1 provides details on the mooring locations associated with the seven buoys used in the analysis (see Fig. 2 ), which were in the vicinity of Hurricane Gordon's track, both in open water and coastal areas around the Canary Islands and Cádiz. All of the instruments measured the wave time series associated with Hurricane Gordon during August 2012.

\section{c. Midresolution wind data}

The study of wave climate conditions requires that wind information be used as forcing within ocean and wave propagation models. In fact, the quality and performance of the wave reanalysis models is conditioned by the quality of the atmospheric surface winds (Reguero et al. 2012), usually measured at $10 \mathrm{~m}$ above mean sea level. This requirement, and the inconvenience of having only both

TABLE 1. Mooring buoy IDs, names, depths, and locations of the seven buoys used for the numerical simulation of Hurricane Gordon and validation.

\begin{tabular}{llccc}
\hline \hline ID & \multicolumn{1}{c}{ Station } & Depth $h(\mathrm{~m})$ & Lat $\left({ }^{\circ} \mathrm{N}\right)$ & Lon $\left({ }^{\circ} \mathrm{W}\right)$ \\
\hline P1 & Sevilla & 10 & 36.740 & 6.480 \\
P2 & Tenerife exterior & 710 & 28.000 & 16.580 \\
P3 & Gran Canaria exterior & 780 & 28.200 & 15.800 \\
P4 & Golfo de Cádiz exterior & 450 & 36.480 & 6.970 \\
P5 & Santa Cruz & 56 & 28.460 & 16.230 \\
P6 & Granadilla & 22 & 28.090 & 16.470 \\
P7 & Coastal Cádiz & 22 & 36.500 & 6.330 \\
\hline
\end{tabular}


sparse and historically limited records measured from buoys, has contributed over the past few years to the incremental use of reanalysis numerical databases. These databases provide homogeneous spatial and temporal records for wind fields all around the globe. This makes them suitable to be used as forcing for any wave generationpropagation model. However, global atmospheric circulation models exhibit low- to midtemporal (between 3 and $6 \mathrm{~h}$ ) and spatial (between $0.7^{\circ}$ and $2.5^{\circ}$ ) resolutions, which does not allow for adequate reproduction of smaller-scale events, such as hurricanes and typhoons (Mínguez et al. 2012).

Currently, the most commonly available global wind reanalysis databases are (i) the 40-yr European Centre for Medium-Range Weather Forecasts (ECMWF) ReAnalysis (ERA-40) database from 1957 to 2002), (ii) the ECMWF Interim Re-Analysis (ERA-Interim) database from 1979 to 2013, (iii) the National Centers for Environmental Prediction (NCEP) and the Climate Forecast System Reanalysis (CFSR) for a 31-yr period (from 1979 to 2009) (Saha et al. 2010), (iv) the Japanese 25-yr Reanalysis (JRA-25) database from 1979 to the present, and (v) the NCEP-National Center for Atmospheric Research (NCAR) reanalysis project (Kalnay et al. 1996), from 1948 up to the present. Each of these databases considers different simplifications to the physical processes involved, as well as different spatial resolutions.

In this study, the 6-h ERA-Interim midresolution $\left(1.5^{\circ} \times\right.$ $1.5^{\circ}$ mesh resolution) wind reanalysis database is selected for three main reasons: (i) it is particularly suitable for users with limited data processing resources, (ii) it is available from the ECMWF Data Server at no charge for research usage, and (iii) it exemplifies the fusion technique between midresolution and high-resolution wind fields (see section 2e), as an essential part of the methodology presented in this study. However, the methodology presented is fully applicable regardless of the midresolution hindcast wind data used.

\section{d. Hurricane high-resolution wind data}

In recent times, most of the studies that numerically have evaluated the generation of waves during hurricane events, such as Smith and Resio (1999), Palmsten and Sallenger (2001), Powell et al. (2010), Bunya et al. (2010), Dietrich et al. (2010, 2011), or Kennedy et al. (2011), have used information from the $\mathrm{H}^{*} \mathrm{WIND}$ database developed by Powell et al. (1998), which provides high-resolution wind and pressure fields for most hurricane events recorded since 1993. This database provides wind fields that can be used, with some adjustments, as forcing for wave propagation and storm surge models.

However, the database does not contain high-resolution wind fields associated with Hurricane Gordon (2012) because the location of this event was beyond the limits of the $\mathrm{H}^{*}$ WIND project. For this reason, in this paper we use wind and pressure fields based on the HydrometRankin Vortex analytical model presented by Holland (1980, 2008) and Bretschneider (1990). This model has already been implemented and validated using data from buoys and satellite-borne synthetic aperture radar during several hurricane historical events, such as in Lehner et al. (2000), Silva et al. (2002), Powell et al. (2010), or Diaz-Hernandez et al. (2012). Figure 3 shows the measured wind speed and pressure time series data (Haeseler 2012) at meteorological stations Ponta Delgada and Santa Maria, located in the Azores Islands, and its comparison with results obtained from the parametric model during Hurricane Gordon. Note the agreement between instrumental measurements and the parametric model.

\section{e. Integration of medium- and high-resolution wind fields}

As discussed in section $2 \mathrm{~b}$, the main drawback of using wind fields from global reanalyses is in their low spatiotemporal resolution, which does not allow us to appropriately reproduce smaller-scale phenomena with a three-dimensional geometry, such as hurricanes. Note that the morphological characteristics (eye, maximum gradient winds, and asymmetry due to its translational speed) of these events are concentrated within a square area not exceeding $100 \mathrm{~km}$ in length. To solve this problem, the present study proposed coupling, integration, and fusion of midresolution wind fields from the ERA-Interim database with high-resolution data obtained from the parametric model. A similar blending technique was used by Chao et al. (2005) to unify Global Forecast System (GFS) and high-resolution wind data from a hurricane prediction model (Geophysical Fluid Dynamics Laboratory (GFDL). The aim here was to adapt forcing data for the SWAN wave propagation model, including more realistic wind fields throughout the numerical domain. For the successful combination of both datasets, spatial identification of the threshold of integration between both data sources is required. This selection guarantees a smooth, continuous, and nonabrupt transition in terms of wind speeds and directions. This task is performed by considering a radial wind decay function, which is applied on the outer edge of the high-resolution parametric wind field:

$$
d=\frac{1}{2} \cos \left(\frac{\pi}{\alpha L} x\right)+\frac{1}{2}
$$

where $\alpha$ is a factor that defines the decaying slope of the wind gradient, $L$ is the radius of the onset threshold decay function measured from the center of the hurricane, and $x$ is the radial distance within $L<x<(\alpha L)$. 

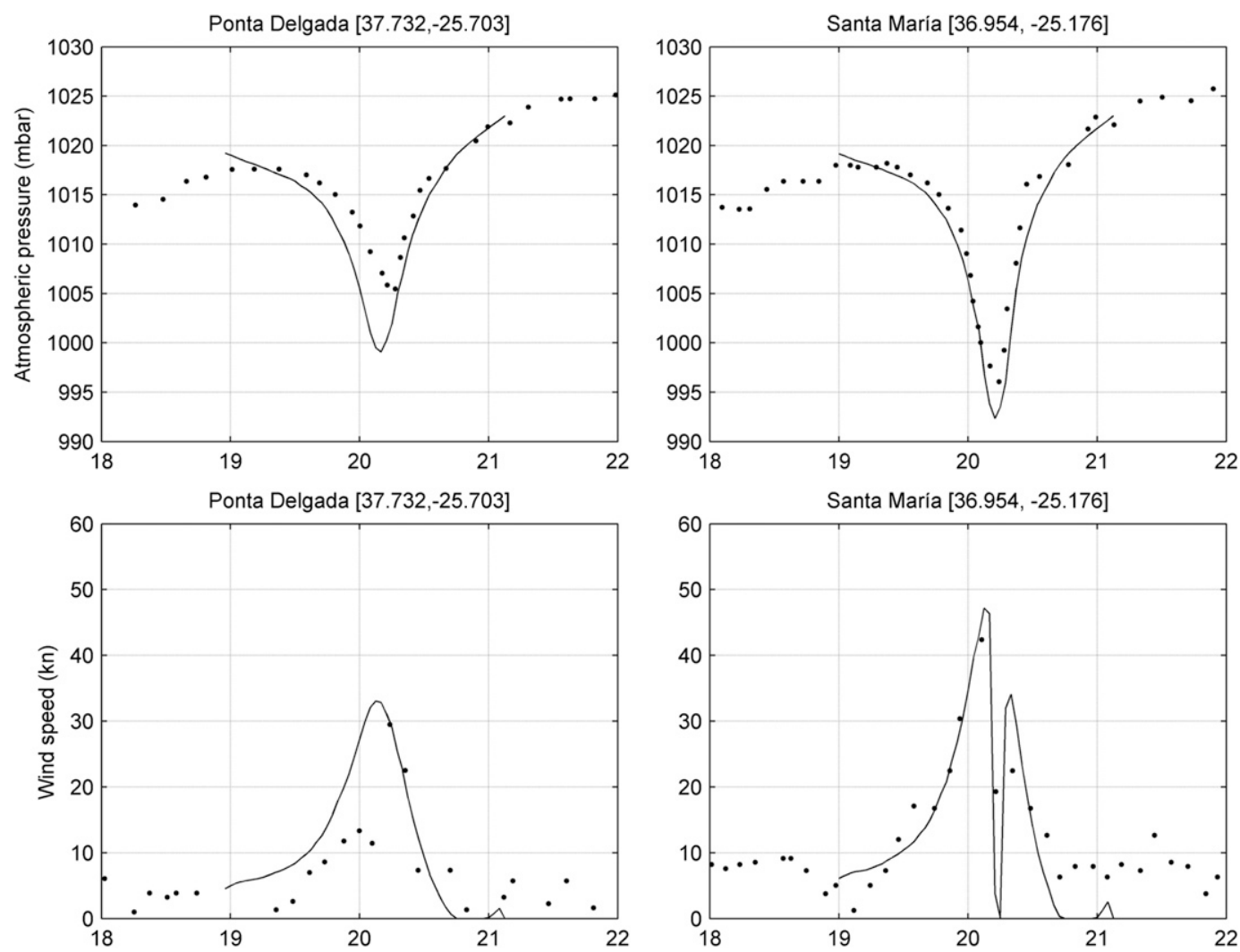

FIG. 3. (top) Pressure and (bottom) wind speed time series obtained from (left) Ponta Delgada and (right) Santa Maria during Hurricane Gordon: weather measurement stations (dots) and the Hydromet-Rankin Vortex model (continuous line).

Figure 4 shows an example of the variation of the decay function presented in this study. This function must be applied radially with respect to the hurricane center position and multiplied by the high-resolution wind field.

After different sensitivity tests and some adjustments to the wind-merging limit, a standard value of $L=5^{\circ}$ was used in this study because it resembles the domain of dimensions $10^{\circ} \times 10^{\circ}$ proposed by the $\mathrm{H}^{*}$ WIND project; and corresponds to the average distance within which wind gradient values commonly decay to $5 \%$ of the maximum hurricane wind speed and are equivalent to the area of influence (AOI) for each storm defined by Chao et al. (2005). Additionally, values of $\alpha$ between $1<\alpha<2$ yield an adequate wind fusion and sufficient numerical stability for the SWAN wave propagation model.

Once high-resolution wind fields are modified by the radial decay function, they can replace the midresolution wind fields. Figure 5 shows an example of the comparative results between the midresolution wind fields for 19 August 2012 at 1100 LT and after merging the highresolution wind field through the decay function.
It is noteworthy that prior to merging both wind field databases, the midresolution database must be interpolated to a finer mesh (e.g., with a spatial resolution of $\Delta x=\Delta y=0.05^{\circ}$ ), and temporarily downscaled to a 1-h time step in order to obtain consistent spatial-temporal homogeneous wind forcing. Additionally, this technique allows quantification of the spectral wave energy associated with hurricanes, by forcing the model with and without the merged high-resolution hurricane wind fields.

Note that we have used a simple parametric model for blending the TC winds because there were no operational wind data for Hurricane Gordon; however, for operational purposes it would be better to use the analyzed and forecast wind from operational forecast centers, such as the operational algorithm designed in the U.S. Navy (Sampson et al. 2010, 2013).

\section{Numerical models SWAN and MOPLA: Description and implementation}

This section briefly describes the equations used in and the implementation of the SWAN (Booij et al. 1999) and MOPLA (González et al. 2007) models, presenting 


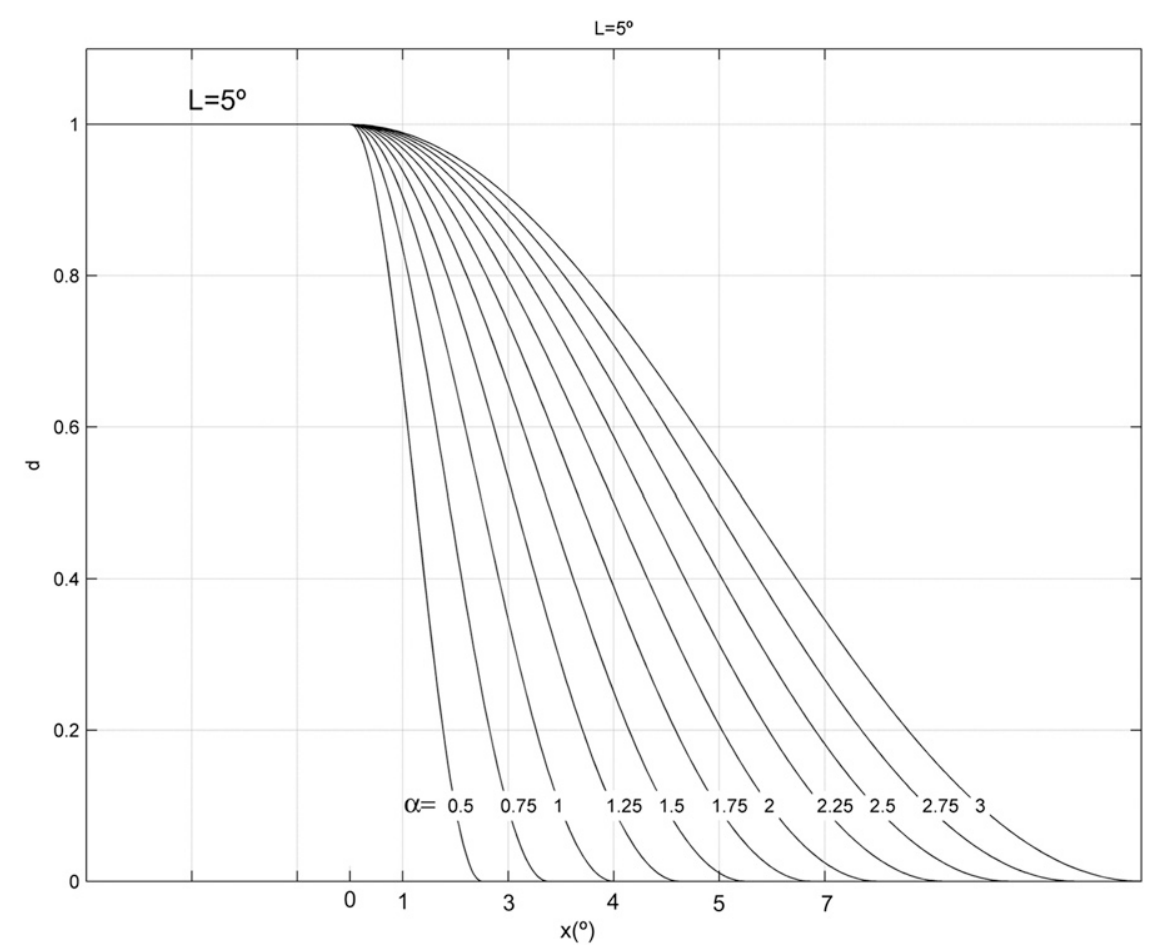

FIG. 4. Radial decay function affecting the high-resolution wind field, for $L=5^{\circ}$ and different values of parameter $\alpha$.

the design of the numerical domains that are based on coastal and bathymetry data.

\section{a. SWAN model}

The SWAN model predicts the evolution in time and space of the wave action spectrum considering the relative frequency and wave direction. It is governed by the balance equation below (Booij et al. 1999):

$$
\frac{\partial N}{\partial t}+\nabla\left[\left(\mathbf{C}_{g}+\mathbf{U}\right) \cdot N\right]+\frac{\partial C_{\theta} N}{\partial \theta}+\frac{\partial C_{\sigma} N}{\partial \sigma}=\frac{S_{\text {tot }}}{\sigma},
$$

where the terms of the left side of (2) correspond to the change in wave action $N$ over time $t$ and spread in space, with $\nabla$ being the gradient operator in the geographic area, $\sigma$ related to the wave period $2 \pi / T, \mathbf{C}_{g}$ the speed of the wave group, and $\mathbf{U}$ the current vector field. The SWAN model is able to reproduce adequately the phenomena of refraction and shoaling along the wave propagation from deep water to the coastal zone by considering the rate of change in the phase $C_{\sigma}$ and spin rate $C_{\theta}$, according to $\partial \sigma$ and $\partial \theta$, respectively (Ris et al. 1999). The term $S_{\text {tot }}$ represents wave growth induced by wind action on the surface of the ocean, including whitecap dissipation, wave breaking, and bottom friction.

In the present study, we have used the unstructured mesh version of the SWAN model by Zijlema (2010), which allows optimizing computational resources. It is noteworthy that the use of SWAN is not recommended for process analysis of wave propagation at a scale smaller than a wavelength (i.e., for higherresolution processes).

To carry out the numerical simulation of Hurricane Gordon, a rectangular domain covering $25^{\circ}-43^{\circ} \mathrm{N}$ and $65^{\circ}-5^{\circ} \mathrm{W}$ was selected. Coastline and bathymetric information was gathered, respectively, from National Oceanic and Atmospheric Administration (NOAA) shoreline-coastline data and the GEBCO database with a resolution of $0.5^{\circ}$. In an attempt to optimize the number of triangular elements associated with the unstructured mesh, without losing the definition of high-resolution hurricane wind fields, the mesh was designed so that it concentrated its elements along the hurricane path, as shown in Fig. 6. The minimum element size all over the hurricane track is approximately $0.05^{\circ}$.

This philosophy of adapting the mesh resolution based on the hurricane track was developed by Popinet et al. (2010). In particular, they used a quadtreeadaptive discretization technique by ensuring the adequate description of the high-resolution hurricane wind fields, which decreased the computational times by between one and two orders of magnitude. 

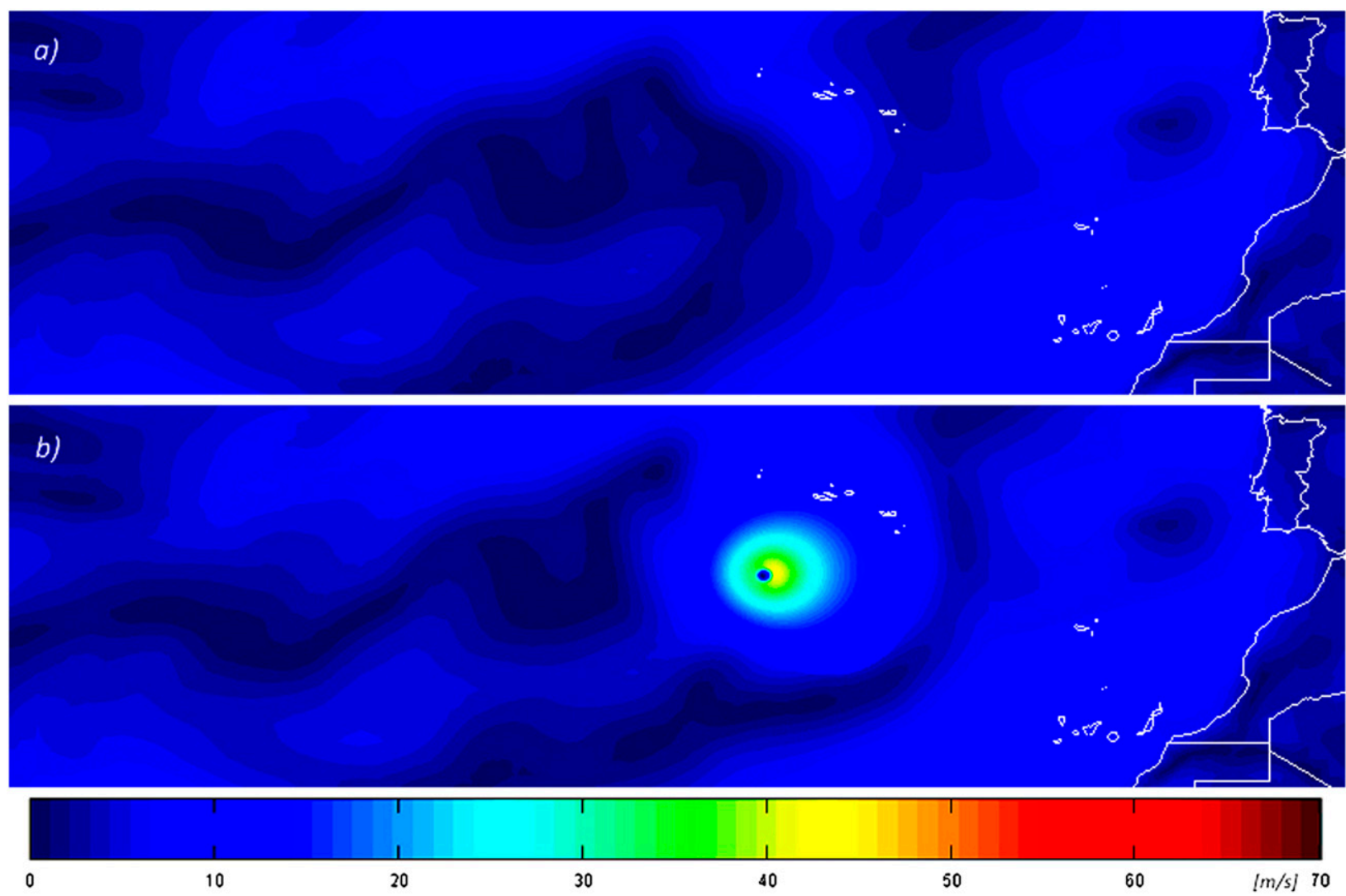

FIG. 5. Example for the fusion work related to wind fields $\left(\mathrm{m} \mathrm{s}^{-1}\right)$ : (a) midresolution (ERA-Interim) and (b) mid- + high-resolution (ERA-Interim + parametric model) at 1100 UTC 19 Aug 2012.

\section{b. MOPLA model}

The MOPLA model (González et al. 2007) is a numerical tool that allows the study of the morphological evolution of coastal areas. It is based on the study of individual processes of waves, currents, and sediment transport in a $2 \mathrm{D}$ horizontal $(2 \mathrm{DH})$ domain. The model consists of three coupled models: the wave transformation model (OLUCA), a vertically averaged wave current module (COPLA), and a sediment transport and morphological evolution module (EROS). In the present study, we have used only the first two models: OLUCA and COPLA.

OLUCA is a phase-averaged wave propagation model that allows the modeling of high-resolution coastal affects (at a resolution not supported by SWAN) such as refraction, shoaling, diffraction, and breaking. The model is capable of considering weakly nonlinear effects

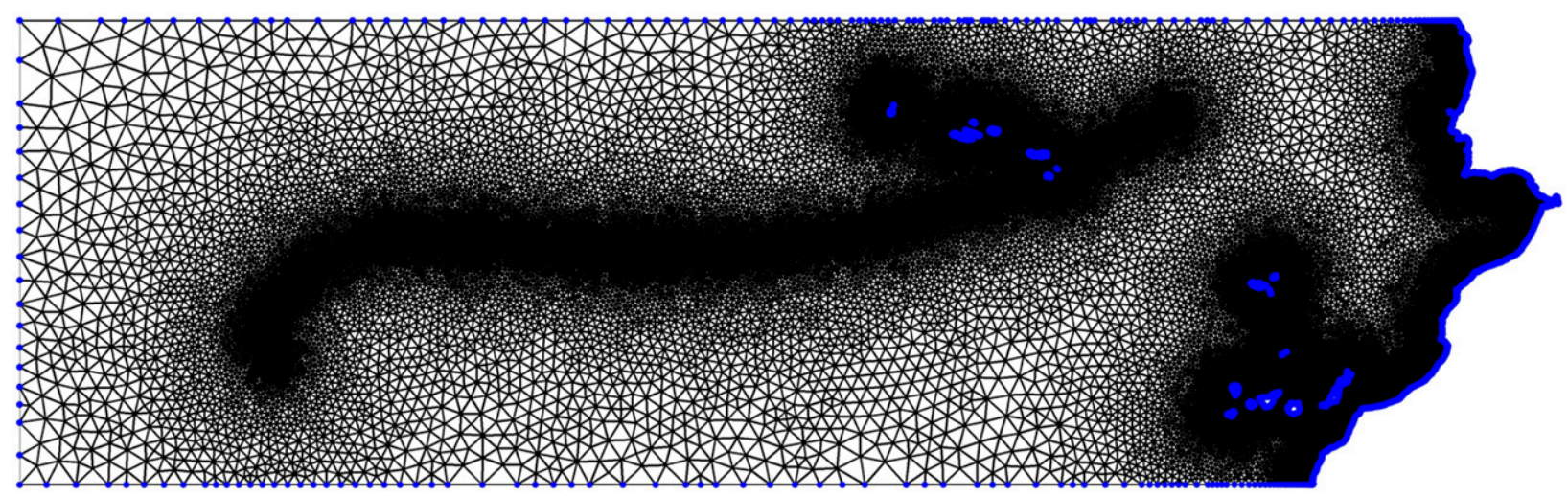

FIG. 6. Spatial domain related to the unstructured triangular mesh for the SWAN model at $25^{\circ}-43^{\circ} \mathrm{N}$ and $65^{\circ}-5^{\circ} \mathrm{W}$. 
associated with shallow waters through the propagation of wave directional spectra over a real bathymetry zone. Additionally, the model includes bottom friction and wave-breaking dissipation effects. It is based on the parabolic approximation of the mild slope equation, initially proposed by Kirby and Dalrymple (1983) and Kirby and Ozkan (1994), and it is implemented in a regular grid under a numerical finite-difference scheme:

$$
\nabla\left(C \times C_{g} \cdot \nabla \eta\right)+\sigma^{2} \frac{C_{g}}{C} \eta=0,
$$

where $C$ is the phase speed of the wave, $C_{g}$ is the group speed, $\sigma$ is the angular frequency, and $\eta$ is the freesurface displacement. The model includes different approximations of surf wave breaking proposed by Battjes and Janssen (1978), Thornton and Guza (1986), and Rattanapitikon and Shibayama (1998). The OLUCA model is an efficient coastal engineering tool because it requires very low computational resources (a regular 4-GB RAM PC).

COPLA solves the vertical integration equations of mass conservation and momentum in the $2 \mathrm{DH}$ field, obtaining nearshore currents induced by irregular breaking waves, through the assimilation of radiation stress maps obtained by the OLUCA model. The model is able to reproduce wave-current interaction, taking into account roughness and turbulent fluctuations (eddy viscosity) related to wave breaking. The two-dimensional COPLA model is derived from the classical NavierStokes equations, which are integrated in the vertical dimension and averaged over a period of time (in the phase of the waves). This scheme uses a coordinate system referenced to the mean sea level. The resulting expressions are the continuity equation,

$$
\frac{\partial U}{\partial t}+\frac{\partial U H}{\partial x}+\frac{\partial V H}{\partial y}=0
$$

and momentum equation with respect to the $x$ component,

$$
\begin{gathered}
\frac{\partial U}{\partial t}+U \frac{\partial U}{\partial x}+V \frac{\partial U}{\partial y}+g \frac{\partial \eta}{\partial x}+\frac{1}{\rho H} \frac{\partial}{\partial}\left(S_{x x}\right)+\frac{1}{\rho H} \frac{\partial}{\partial}\left(S_{y y}\right) \\
+\frac{g U}{C^{2} H}\left(U^{2}+V^{2}\right)^{1 / 2}-\epsilon\left(\frac{\partial^{2} U}{\partial x^{2}}+\frac{\partial^{2} V}{\partial y^{2}}\right)=0,
\end{gathered}
$$

where $H=\eta+h$ is the depth (free surface $\eta$ and depth $h), U$ is the velocity component in the $x$ dimension, $V$ is the velocity component in the $y$ dimension, $S_{x x}$ and $S_{y y}$ are the radiation stresses in each direction, $\rho$ is the water density, $g$ is the gravitational acceleration constant, $C$ is the Chezy friction parameter, and $\epsilon$ is the eddy viscosity term.
Bathymetry maps were digitized and their geographic coordinates converted into metric coordinates with respect to the Mercator projections in the 1984 revision of the World Geodetic System (WGS84), universal transverse Mercator (UTM), for $30^{\circ}$ and $29^{\circ} \mathrm{S}$. This also includes detailed bathymetry information for the beaches of La Barrosa, Conil, El Palmar, Zahara, Barbate, and Zahara in Cádiz.

Figure 7 shows (i) the bathymetry integration of different databases on the same mesh, interpolated for a resolution of $25 \mathrm{~m} \times 25 \mathrm{~m}$, (ii) the four meshes designed for the OLUCA and COPLA models, and (iii) the location of the corresponding wave coupling points. Note that topography and bathymetry points are referenced with respect to the average of the lowest spring tides.

\section{Numerical wave propagation associated with Hurricane Gordon}

\section{a. Introduction}

The third generation of the SWAN model (GEN3) (Janssen 1989) for wave growth model forced by wind action is used because, as mentioned in Huang et al. (2013), it provides better results for stronger winds above $100 \mathrm{~km} \mathrm{~h}^{-1}$ in comparison with the approach by Komen and Hasselmann (1984). The simulation performed with the SWAN model provides output files as maps of $H_{s}, T_{p}$, $\theta$, wind speed $W$, and wind direction $\beta$, as well as wave spectra at any point within the numerical domain. Section $4 \mathrm{~b}$ presents $2 \mathrm{DH}$ map results, which diagnose the behavior of waves and wind in geographic and temporal domains during the hurricane event. Section $4 \mathrm{c}$ presents time series graphs of wave parameters obtained with the SWAN model simulation compared with buoy data. Finally, section $4 \mathrm{~d}$ analyzes and discusses the changes undergone by the wave spectra off the coast of Cádiz, through the direct comparison of both alternatives, i.e. with and without high-resolution wind field merging.

\section{b. Simulation of Hurricane Gordon (2012)}

A SWAN model simulation was performed using a nonstationary wind forcing, which reproduces the creation, evolution, propagation, and dissipation of Hurricane Gordon from 10 until 28 August 2012. A time step of $10 \min (\Delta t=10)$ was selected to ensure the stability and convergence of the model.

Figure 8 shows the evolution of significant wave heights $\left(H_{s}\right)$ and peak periods $\left(T_{p}\right)$ at different times. Note how $H_{s}$ evolves as Hurricane Gordon moves, reaching a maximum value of $H_{s}=12 \mathrm{~m}$ in the area adjacent to the track of the hurricane. Fortunately, this extreme, significant wave-height increment near the 


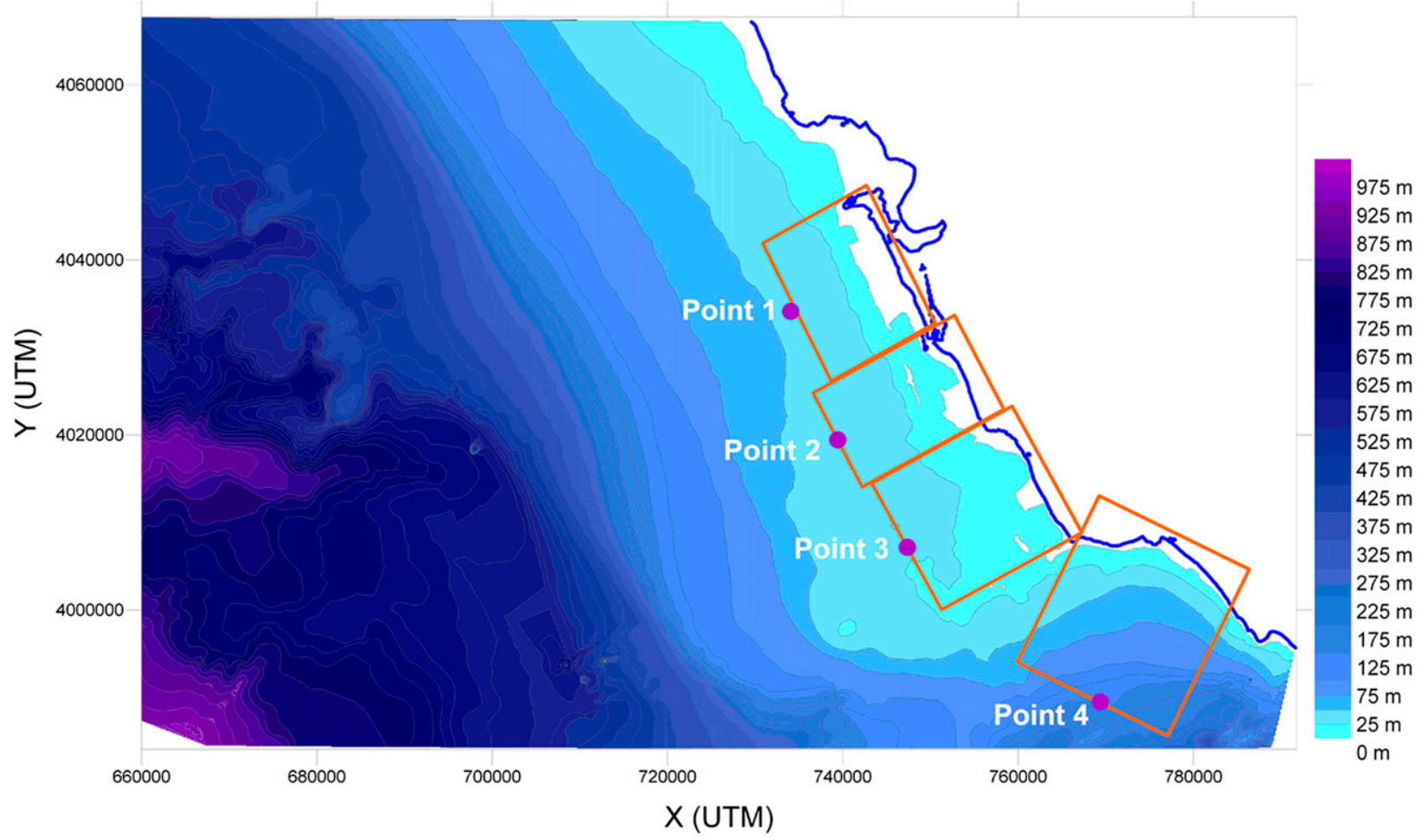

FIG. 7. Bathymetry and numerical meshes used by the MOPLA model for analysis of waves and currents along the coast of Cádiz. Points 1-4 correspond to wave coupling locations.

center of the hurricane does not reach the coastal area of Cádiz; only a low-energy swell $H_{s}<2 \mathrm{~m}$ reaches the Gulf of Cádiz.

Regarding the peak-period evolution, Fig. 8 also shows the translation of the hurricane toward the east, where the peak period increased considerably. It is worth noting that from 20 August 2012, the growth of peak periods propagated toward the Gulf of Cádiz, where the presence of periods above $T_{p}>12 \mathrm{~s}$ is evident, and finally reached the coast of Cádiz. After this time, peak periods decreased slowly from 21 to 24 August 2012 to the usual peak periods in the Gulf of Cádiz of around $T_{p}=5 \mathrm{~s}$.

\section{c. Validation of numerical data}

In this section, the validation of the SWAN model in terms of $H_{s}$ and $T_{p}$ is performed by comparing its numerical results with instrumental data from ocean buoys. As discussed in section 2, seven time-series measurements from buoys located within the the Canary Islands and in the Gulf of Cádiz were obtained. All buoys provide continuous records of hourly information associated with wave spectral parameters, except for the Gulf of Cádiz buoy, which only measured wave data during 24 August 2012.
Figures 9 and 10 show the time series of $H_{s}$ and $T_{p}$ modeled and measured at the selected stations in the Canary Islands and the Gulf of Cádiz areas, respectively. Coefficients of determination $\left(R^{2}\right)$ were calculated for all the buoys with values between 0.57 and 0.92 for $H_{s}$, between 0.63 and 0.99 for $T_{p}$, and of 0.92 for the mean wave direction at the Cádiz buoy (regardless of the series, which have few points of comparison).

Results from Fig. 9 show the numerical representation of the significant wave height and its evolution over time for the four stations located in the Canary Islands. In this zone, the possible influence of the hurricane is practically nonexistent because $H_{s}$ remains around $1.5 \mathrm{~m}$.

With regard to the possible influence of the hurricane on the peak period in the Canary Islands, the general trend provided by the numerical model is shown to be appropriate. However, one can note that for the Gran Canaria and Santa Cruz buoys, the instruments recorded increases in $T_{p}$ beginning on 16 August 2012, especially at the Gran Canaria buoy. This sudden increase in $T_{p}$ is due to the influence of the swell generated by the hurricane. However, SWAN is not able to reproduce such increases, probably because it is unable to properly manage the wave front turning due to the diffraction from the island of Tenerife, which acts as a natural 

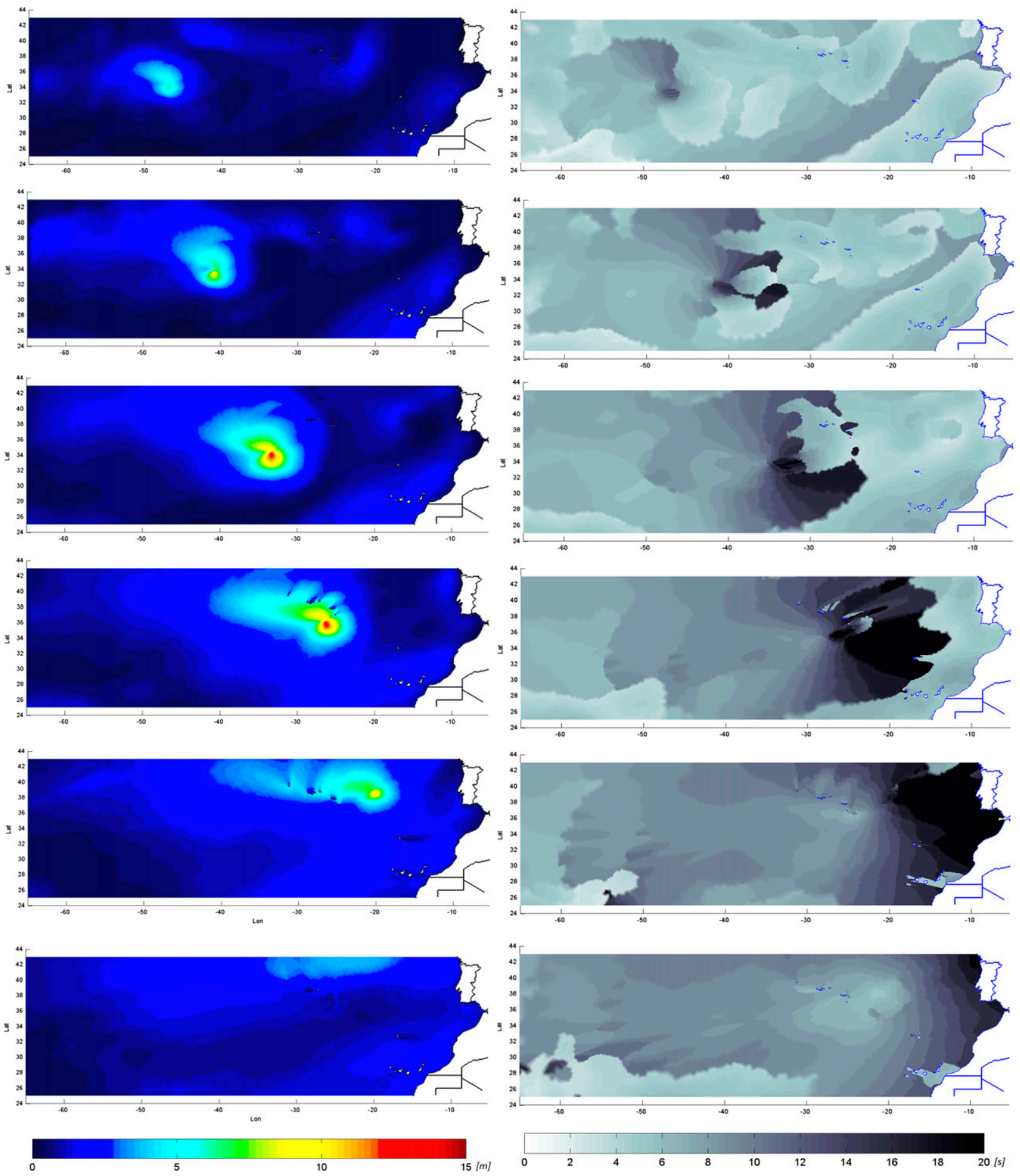

FIG. 8. Evolution of (left) $H_{s}$ and (right) $T_{p}$ for Hurricane Gordon obtained with the SWAN model for (from top to bottom) September 2012 (hour in UTC/day): 1200/17, 0800/18, 0900/19, 0000/20, 2000/20, and 1600/21. The map coordinates run from $24^{\circ}$ to $44^{\circ} \mathrm{N}$ and from $10^{\circ}$ to $65^{\circ} \mathrm{W}$ and the color scales from 0 to $15 \mathrm{~m}$ and from 0 to $20 \mathrm{~s}$.

blocking element for waves coming from the northwest, and provides shelter for the area between Tenerife and the island of Gran Canaria, which is precisely where these two buoys are located. This limitation of the SWAN model is well known and is discussed in the model development documents (Zijlema 2010). This shadow effect can be perfectly seen in Fig. 11, where the position of the outer Gran Canaria buoy is also shown (red dot). 

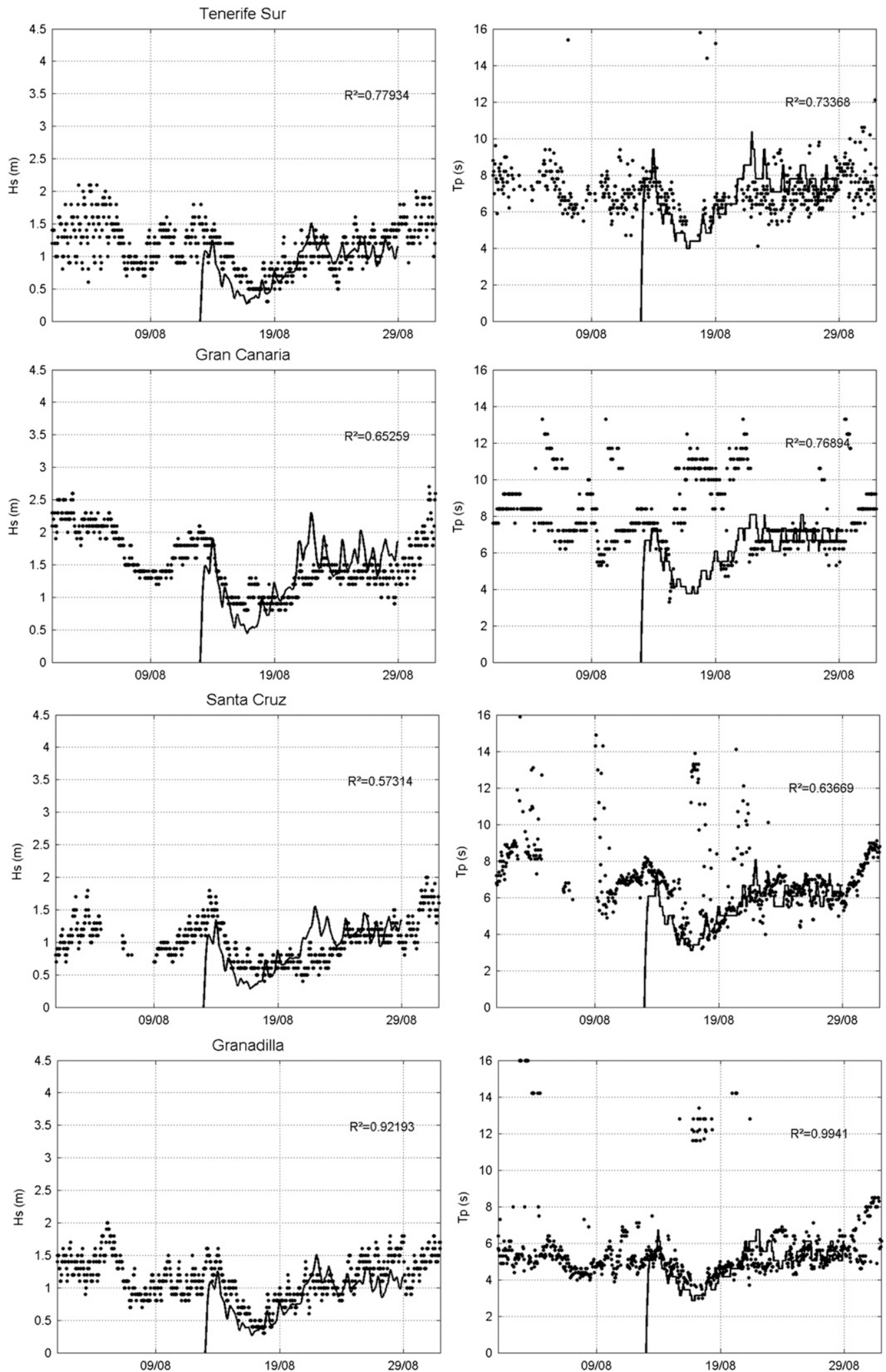

FIG. 9. Validation results for (left) $H_{s}$ and (right) $T_{p}$ for the SWAN model (continuous line) and the buoy instrumental data (dots) from the Canary Islands for 1-30 Aug ( $x$ axis): (from top to bottom) Tenerife Sur, Gran Canaria, Santa Cruz, and Granadilla. The $y$ axis runs from 0 to $4.5 \mathrm{~m}$ for $H_{s}$ and from 0 to $16 \mathrm{~s}$ for $T_{p}$. The coefficient of determination $\left(R^{2}\right)$ is given in each panel. Dates are in day/month format. 

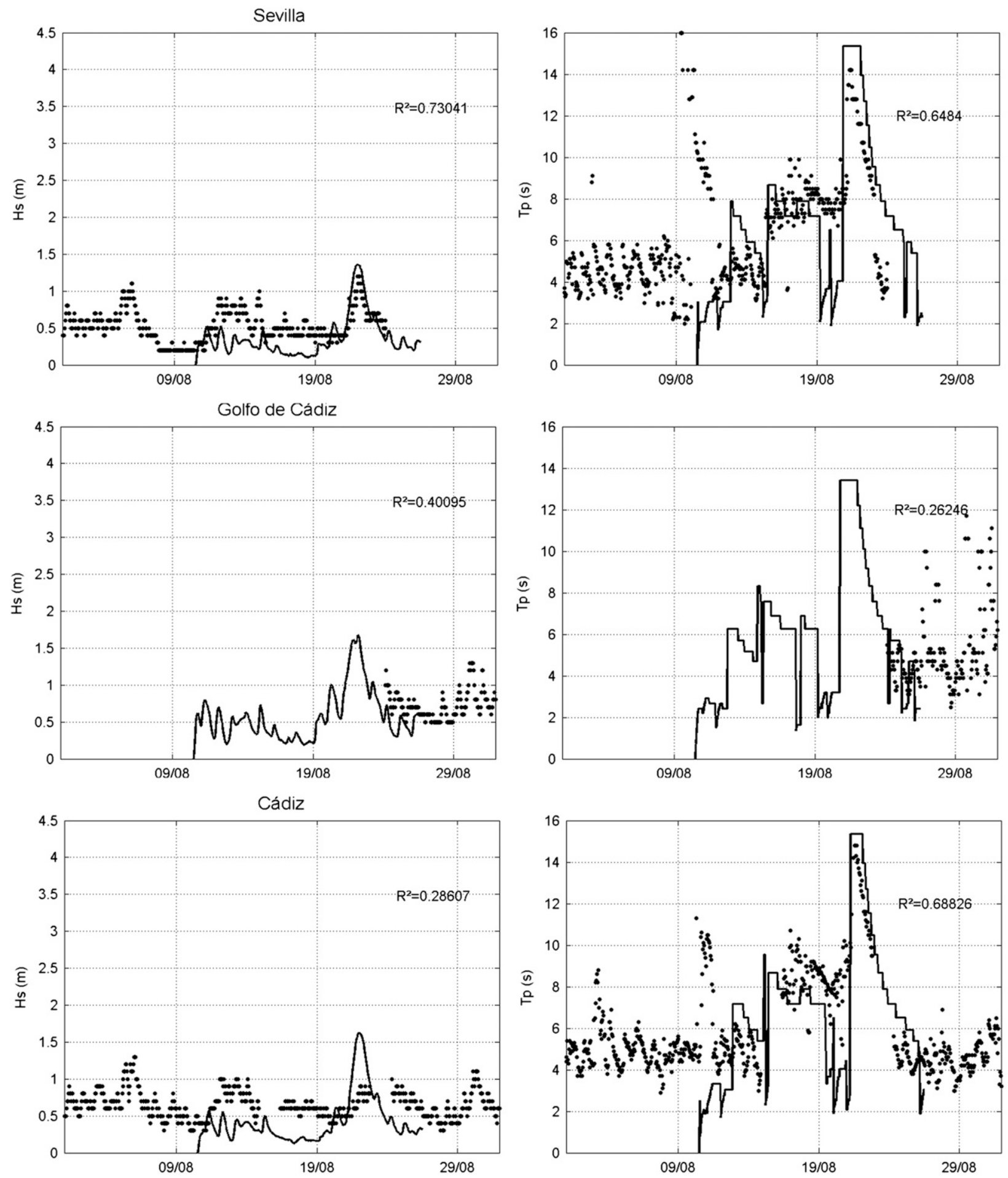

FIG. 10. As in Fig. 9, but for (from top to bottom) Sevilla, Golfo de Cádiz, and Cádiz with buoy instrumental data from the Gulf of Cádiz.

Figure 10 shows the wave evolution for the Seville and Cádiz buoys. The SWAN model is able to follow the evolution of $H_{s}$ for Hurricane Gordon, showing magnitude increases from 19 August, which reach a maximum of $1.5 \mathrm{~m}$ that agrees with the buoy measurements. We can conclude that the energy contribution of Hurricane Gordon to the Gulf of Cádiz occurred around $H_{s}=1 \mathrm{~m}$, because, as shown in Fig. 10, the $H_{s}$ values before and 

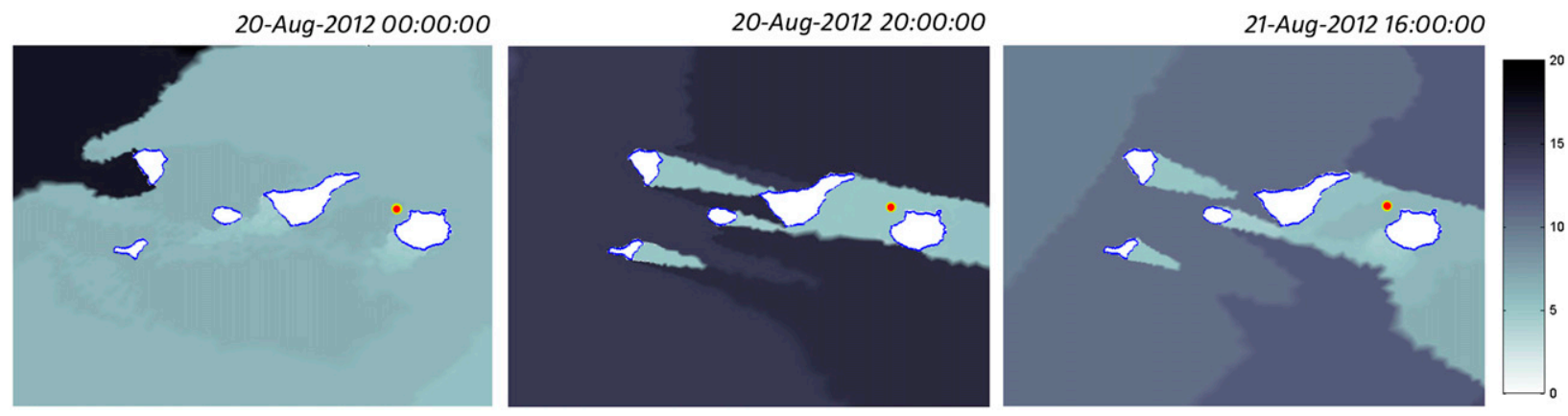

FIG. 11. Example of peak period (s) spatial spread in the Canary Islands during Hurricane Gordon with data from the Gran Canaria buoy (red dot): (left) 0000 UTC 20 Aug, (middle) 2000 UTC 20 Aug, and (right) 1600 UTC 21 Aug 2012.

after the event were about $0.5 \mathrm{~m}$, which coincides with the observations from civil protection, local police, and Red Cross personnel (Aparicio Florido et al. 2013).

Regarding the evolution of the peak period for the same dates, the buoy records register a sharp rise in peak periods $>12 \mathrm{~s}$, reaching a maximum of $T_{p}=14 \mathrm{~s}$ during 21 August 2012. This sudden increase reached its maximum within a few hours and dropped finally to normal values (of around $T_{p}=5 \mathrm{~s}$ ) 3 days later. This behavior is well reflected by the SWAN model, which shows an even more abrupt change than that recorded by the buoys, but manages to reproduce the generation of the lowenergy swell sea state observed in the Gulf of Cádiz. Finally, Fig. 12 shows the validation of the mean wave propagation direction related to the Cádiz buoy, where westerlies are the predominant direction toward the coast of Cádiz prior to Hurricane Gordon. A sudden change of $\theta=60^{\circ}$ to the south occurs due to the hurricane with a normalization of the mean conditions during the three following days.

\section{d. Analysis of the spectral changes due to Hurricane Gordon}

This section shows the analysis of the spectral information recorded by the numerical model at the Cádiz buoy (point 3 in Fig. 7), which corresponds to the coupling point between the SWAN and MOPLA models. Point 3 is located at $36.131^{\circ} \mathrm{N}, 6.454^{\circ} \mathrm{W}$, over a depth of approximately $30 \mathrm{~m}$. Wave data at this point are considered representative of the wave climate in the intermediate waters of the Gulf of Cádiz. As mentioned previously, the SWAN model provides nonstationary directional energy spectra before and after the hurricane event. These wave spectra data at point 3 are used to force the MOPLA model for the numerical evaluation of hydrodynamics in the surf zone. To evaluate the temporal evolution of the swell and growth in peak periods produced by the hurricane, Fig. 13 shows the temporal evolution of the spectral information (integrated along the directional dimension) at point 3 for two different SWAN model simulations: (i) taking into account wind forcing of midresolution (ERA-Interim) + highresolution wind (vortex model) and (ii) considering only medium-resolution (ERA-Interim) data (i.e., without including the presence of the hurricane). Figure 13a shows a significant increase in the wave spectrum toward higher periods, reaching a limit of $T_{p}=14 \mathrm{~s}$ with a clear energy concentration that dissipates or diminishes over the next 3-4 days after the hurricane.

\section{Numerical propagation of waves and currents in the coastal zone of Cádiz}

\section{a. Introduction}

This section presents the numerical analysis of hydrodynamics in the surf zone along the coast of Cádiz by analyzing the behavior of waves and currents on the

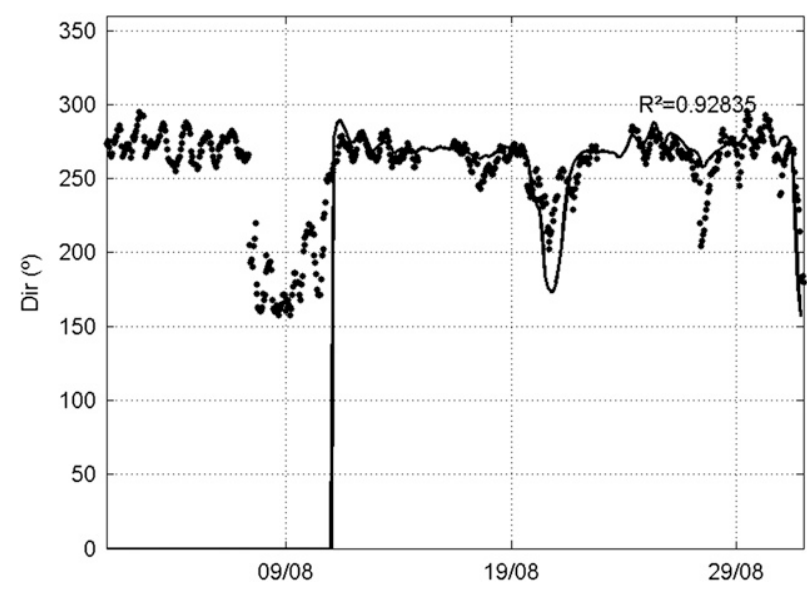

FIG. 12. Evolution of the average direction of wave propagation $\theta$ at the Cádiz coastal buoy (dots) and its comparison with the SWAN model results (line) for 1-30 Aug ( $x$ axis). The coefficient of determination $\left(R^{2}\right)$ is also shown. 

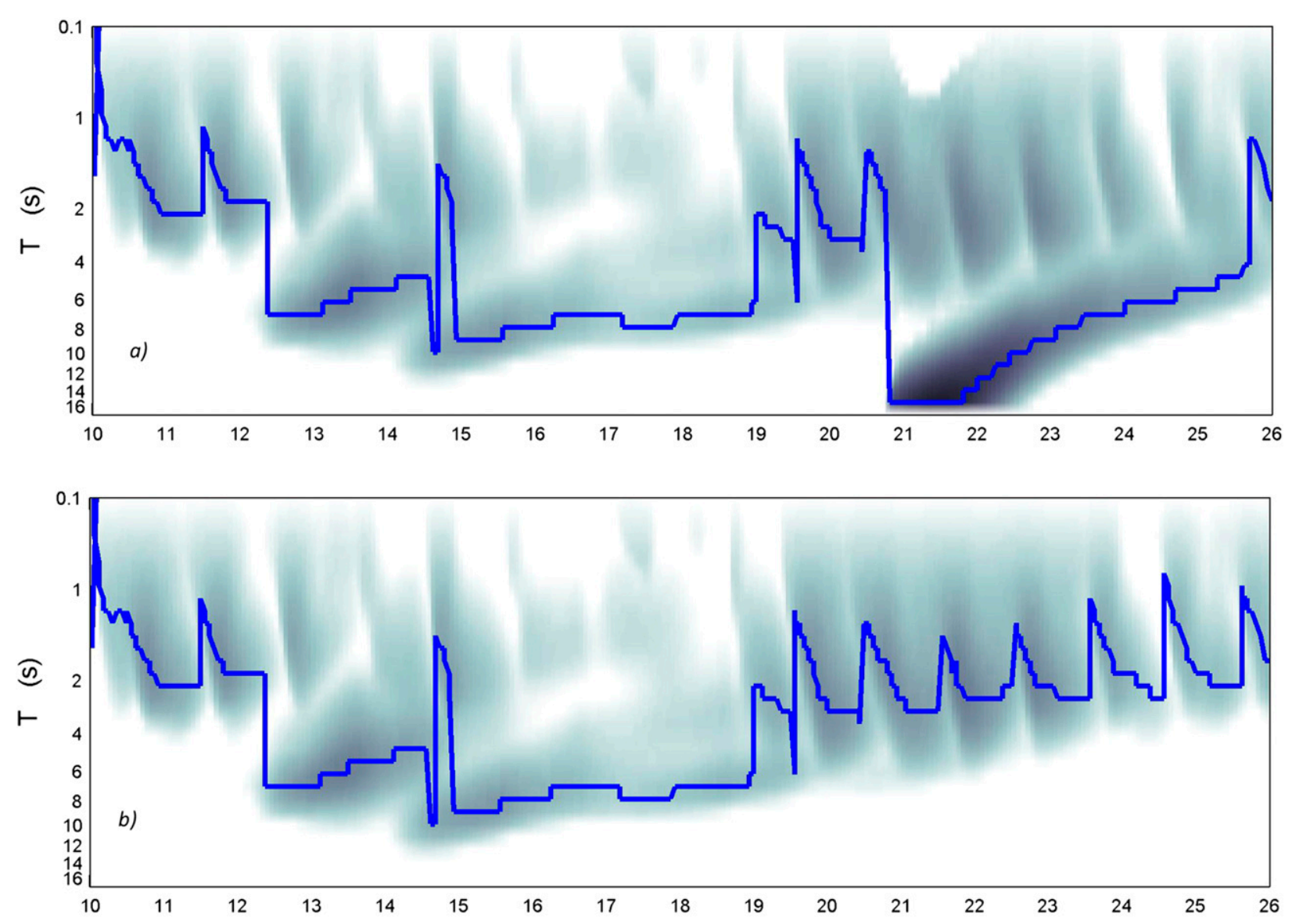

August 2012

FIG. 13. The 10-26 Aug 2012 evolution of the energy spectrum of periods (shading, s) and the peak period (blue line, s) at location $3\left(36.131^{\circ} \mathrm{N}, 6.454^{\circ} \mathrm{W}\right)$ for (a) mid- + high-resolution winds and (b) only midresolution winds without the inserted vortex.

beach, before and after Hurricane Gordon. Two numerical models were used for this task: the nearshore wave propagation model (MOPLA) and the wavebreaking currents model (COPLA). Figure 7 shows the limits of the numerical meshes designed used for the propagation of waves toward the coastal area. These take into consideration (i) the mean wave direction recorded during the second half of August, (ii) the coastline continuity, and (iii) the maximum resolution of the mesh based on the computational limitations of the model. Their spatial resolution is $\Delta x=\Delta y=50 \mathrm{~m}$ and the initial depth of the wave propagation was taken around $h=40 \mathrm{~m}$. The simulations were forced with hourly spectral wave time series obtained from the SWAN model at four control points (shown in Fig. 7). We took into consideration sea level variations by reconstructing the astronomical tide using data from the Bonanza tide gauge $\left(36.80^{\circ} \mathrm{N}, 6.34^{\circ} \mathrm{W}\right)$. A total of 332 sea states, from 1700 UTC 12 August to 1200 UTC
26 August) were simulated, obtaining the evolution of waves and currents all along the coastline.

Note that this analysis has not been quantitatively validated due to the lack of measurements along the coast. However, a qualitative analysis was performed based on the report submitted by Aparicio Florido et al. (2013), where a catalog of oblique aerial photographs was collected during emergency events recorded in the beach in Conil de la Frontera (provided by civil protection personnel).

\section{b. Results of waves and currents at coast}

For comparison purposes, Fig. 14 shows a spatial integration of maps of currents along the Cádiz coastline at two different instants: (i) just before the hurricane event and (ii) after the arrival of the swell sea state induced by Hurricane Gordon. It can be seen that the currents before the arrival of the 20 August 2012 event did not exceed $0.05 \mathrm{~m} \mathrm{~s}^{-1}$, which can be considered safe for bathing. However, when the swell associated with 


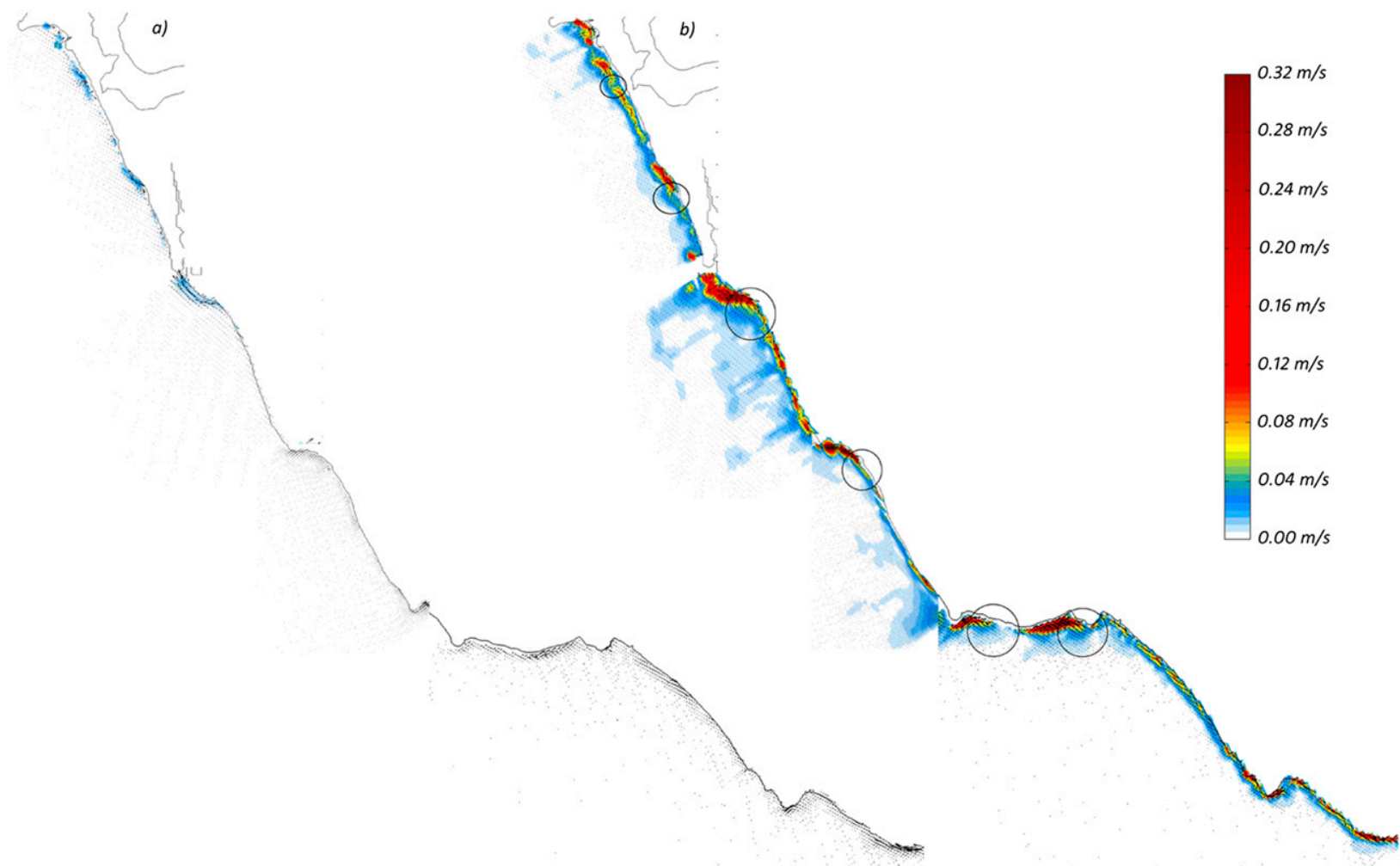

FIG. 14. Map of currents along the coast of Cádiz at two different instants: (a) prior the event of 1000 UTC 20 Aug and (b) after arrival of the swell induced by Hurricane Gordon on 1000 UTC 21 Aug 2012. The current scale runs from 0.00 to $0.32 \mathrm{~m} \mathrm{~s}^{-1}$.

higher periods reached the coast of Cádiz, the currents induced by wave breaking along the coast underwent a dramatic increase, reaching average hourly longitudinalcurrent values $>0.5 \mathrm{~m} \mathrm{~s}^{-1}$, and maximum instantaneous values close to $1.0 \mathrm{~m} \mathrm{~s}^{-1}$ in certain zones. It should be noted the presence of rip currents (indicated by black circles in Fig. 14) is represented by the change in wave direction shown in Fig. 12. These values are consistent

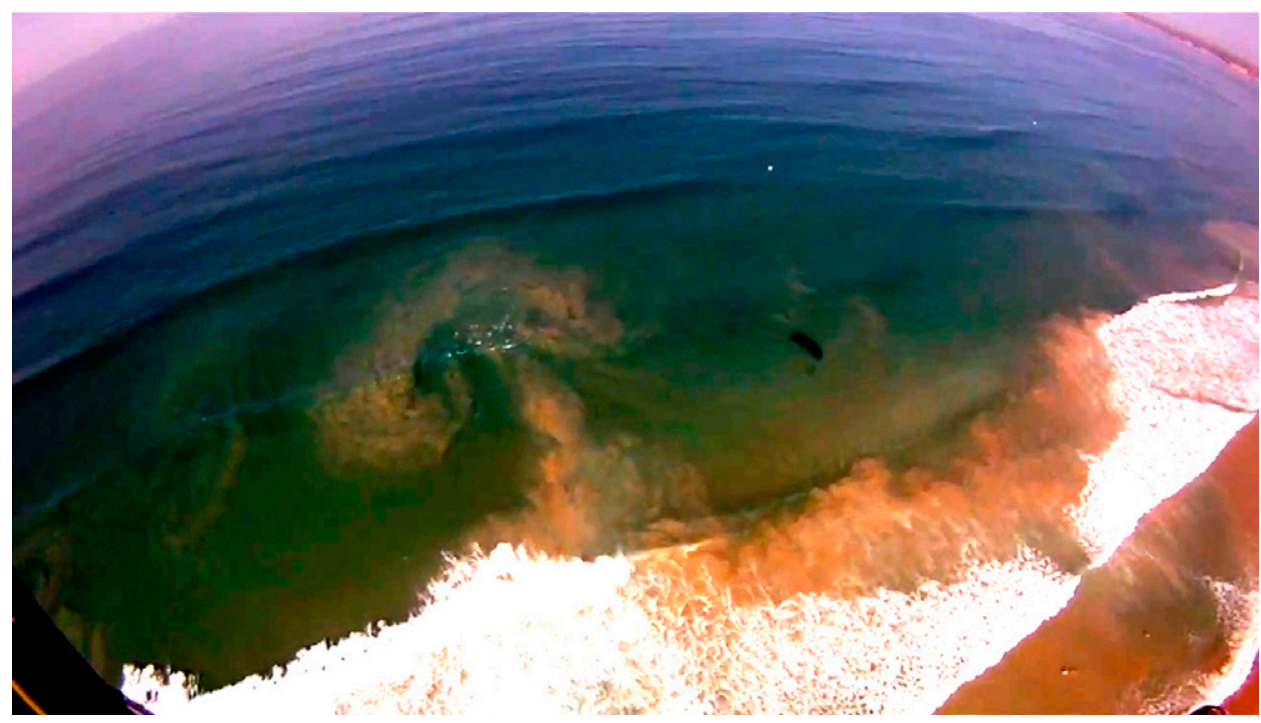

FIG. 15. Picture of a rip current observed along the coast of Conil de la Fontera during a swell event that occurred on 21 Aug 2012. (Source: Civil Protection Conil de la Fontera.) 

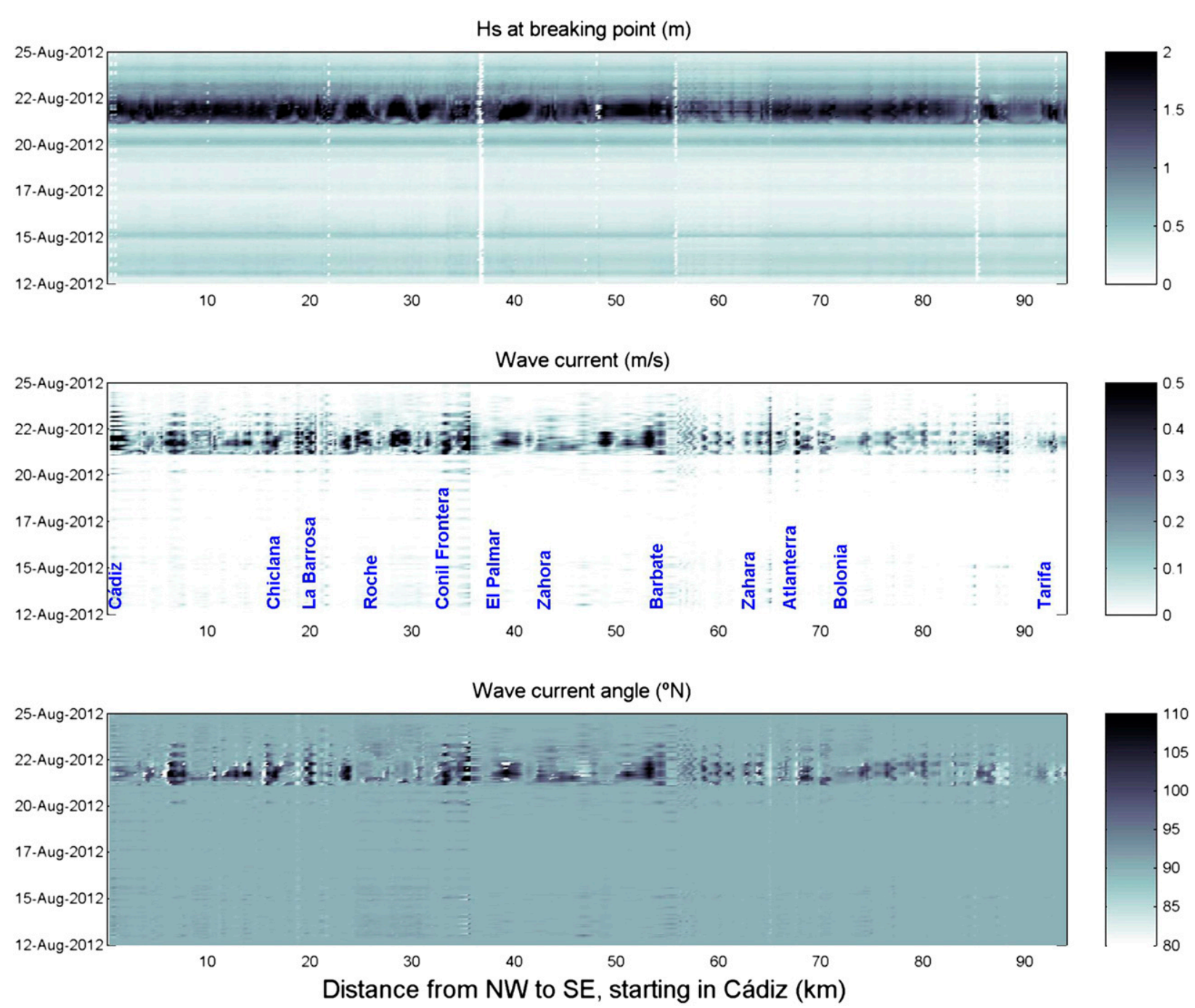

FIG. 16. Spatial-temporal evolution of (top) significant wave height ( $\mathrm{m}$ ) at the breaking point, (middle) the current velocity ( $\mathrm{m} \mathrm{s}{ }^{-1}$ ), and (bottom) the average direction $\left({ }^{\circ} \mathrm{N}\right)$ of the current along the coast of Cádiz during Hurricane Gordon.

with the observations of civil protection personnel at Conil de la Frontera city (see photo in Fig. 15), where the safety for bathing was clearly compromised. This sort of analysis could be easily used to restrict swimming at the beach, following the operational guidance for safe European beaches published by the Royal Society for the Prevention of Accidents (RoSPA 2005).

Finally, Fig. 16 shows the temporal and spatial evolution of (i) significant wave heights at the breaking point, (ii) the current velocity, and (iii) the average direction of the current during the event. In the top panel, one can see that the change in $H_{s}$ produced by the swell created by Hurricane Gordon is about $2 \mathrm{~m}$, which corresponds to breaking-limit conditions. Second, it can also be observed that the current speed and angle (Fig. 16 , middle and bottom) show well-defined packets of high-energy currents along the coast of Cádiz with speeds above $0.5 \mathrm{~m} \mathrm{~s}^{-1}$ associated with abrupt changes of the wave current angle from $50^{\circ}$ to $150^{\circ}$. This is a clear indication of the presence of high-energy rip currents.

\section{Conclusions}

This study reproduces the generation, propagation, and interaction at the coast of sea states generated by Hurricane Gordon during the second half of August 2012. The study involved the coupling of different analytical and numerical tools and databases of different spatial and temporal scales. It shows the usefulness of using parametric models to provide an adequate definition of high-resolution winds associated with hurricanes similar to Gordon, and it presents an easy-to-use 
technique for its integration with midresolution (ERAInterim) wind fields.

The SWAN model has proven to be a versatile and reliable tool for performing studies on the generation and propagation of waves associated with hurricane events, specifically for: (i) conducting a nonstationary run, (ii) using adaptive and irregular meshes, (iii) including high-resolution wind fields, and (iv) using real geographic and bathymetry charts.

The study demonstrated that coupling the SWAN and MOPLA models for wave propagation in the surf zone is possible. This allows us to obtain the magnitude and direction of flow patterns associated with hurricane waves breaking toward the coast.

Regarding the Hurricane Gordon event, we conclude that it produced waves of $H_{s}=1.5 \mathrm{~m}$ and $T_{p}=14 \mathrm{~s}$ in an unusual and sudden span of a few hours. These sea state conditions interacted with the coast of Cádiz, inducing strong currents $\left(>0.5 \mathrm{~m} \mathrm{~s}^{-1}\right)$ and dangerous rip currents that compromised the safety of bathers.

This paper provides the appropriate framework for civil protection agencies, especially those located in any geographical area where hurricane events occur, to implement an operational system coupled with early hurricane warning systems. This methodology can be adapted as an operational system of hurricane effects along any coastline. This information is crucial for making informed decisions about safety plans, coastline warning systems, bathing limitations, and responses to cases of emergency situations.

Acknowledgments. We thank Puertos del Estado (Spain) for the access to buoy information and the International Association of Emergency Managers (IAEM) for its technical support and for providing the photographic information collected by the Red Cross and Civil Protection along the coast of Cádiz. This work was partially funded by projects "GRACCIE" (SD 200700067, CONSOLIDER INGENIO 2010) and "iMar21" (BiA2011-2890) from the Spanish government.

\section{REFERENCES}

Aparicio Florido, J. A., and Coauthors, 2013: La resaca marina del 21 de agosto de 2012 en las costas de Cádiz. IAEM-España, $86 \mathrm{pp}$. [Available online at http://www.iaem.es/Documentos/ informe-resacamarina-cadiz-21082012.pdf.]

Avila, L. A., 2012: Tropical cyclone report: Hurricane Gordon (AL082012) 15-20 August 2012. National Hurricane Center, $12 \mathrm{pp}$. [Available online at http://www.nhc.noaa.gov/data/tcr/ AL082012_Gordon.pdf.]

Battjes, J., and J. Janssen, 1978: Energy loss and set-up due to breaking of random waves. Coastal Engineering 1978: Proceedings of the 16th Coastal Engineering Conference, B. L. Edge, Ed., American Society of Civil Engineers, 569-587.
Booij, N., R. C. Ris, and L. H. Holthuijsen, 1999: A thirdgeneration wave model for coastal regions: 1 . Model description and validation. J. Geophys. Res., 104, 7649-7666, doi:10.1029/98JC02622.

Bretschneider, C., 1990: Tropical cyclones. Handbook of Coastal and Ocean Engineering, J. B. Herbich, Ed., Gulf Publishing, 249-370.

Bunya, S., and Coauthors, 2010: A high-resolution coupled riverine flow, tide, wind, wind wave, and storm surge model for southern Louisiana and Mississippi. Part I: Model development and validation. Mon. Wea. Rev., 138, 345-377, doi:10.1175/ 2009MWR2906.1.

Chao, Y., J.-E. G. M. Alves, and H. L. Tolman, 2005: An operational system for predicting hurricane-generated wind waves in the North Atlantic Ocean. Wea. Forecasting, 20, 652-671, doi:10.1175/WAF851.1.

Diaz-Hernandez, G., L. Robles, C. Appendini, F. Mendez, A. Torres-Freyermuth, I. Losada, and S. P., 2012: An engineering approach for modeling hurricane extreme waves using analytical and numerical tools. Proc. ATC and SEI Conf. on Advances in Hurricane Engineering, Miami, FL, American Society of Civil Engineers, 765-776, doi:10.1061/9780784412626.066.

Dietrich, J. C., and Coauthors, 2010: A high-resolution coupled riverine flow, tide, wind, wind wave, and storm surge model for southern Louisiana and Mississippi. Part II: Synoptic description and analysis of Hurricanes Katrina and Rita. Mon. Wea. Rev., 138, 378-404, doi:10.1175/2009MWR2907.1.

— storm surge: Hindcast, synoptic analysis, and validation in southern Louisiana. Mon. Wea. Rev., 139, 2488-2522, doi:10.1175/2011MWR3611.1.

González, M., R. Medina, J. Gonzalez-Ondina, A. Osorio, F. J. Méndez, and E. García, 2007: An integrated coastal modeling system for analyzing beach processes and beach restoration projects, SMC. Comput. Geosci., 33, 916-931, doi:10.1016/ j.cageo.2006.12.005.

Haeseler, S., 2012: Hurricane Gordon in the area of the Azores in August 2012. Deutscher Wetterdienst Rep., 6 pp.

Holland, G., 1980: An analytic model of the wind and pressure profiles in hurricanes. Mon. Wea. Rev., 108, 1212-1218, doi:10.1175/1520-0493(1980)108<1212:AAMOTW>2.0.CO;2.

_ 2008: A revised hurricane pressure-wind model. Mon. Wea. Rev., 136, 3432-3445, doi:10.1175/2008MWR2395.1.

Huang, Y., R. H. Weisberg, and L. Zheng, 2013: Gulf of Mexico hurricane wave simulations using SWAN: Bulk formula based drag coefficient sensitivity for Hurricane Ike. J. Geophys. Res. Oceans, 118, 3916-3938, doi:10.1002/jgrc.20283.

Janssen, P. A. E. M., 1989: Wave-induced stress and the drag of air flow over sea waves. J. Phys. Oceanogr., 19, 745-754, doi:10.1175/1520-0485(1989)019<0745:WISATD>2.0.CO;2.

Jarvinen, B. R., C. J. Neumann, and M. A. S. Davis, 1984: A tropical cyclone data tape for the North Atlantic basin, 18861983: Contents, limitations, and uses. NOAA Tech. Memo. NWS 22, 24 pp. [Available online at http://www.nhc.noaa.gov/ pdf/NWS-NHC-1988-22.pdf.]

Kalnay, E., and Coauthors, 1996: The NCEP/NCAR 40-Year Reanalysis Project. Bull. Amer. Meteor. Soc., 77, 437-471, doi:10.1175/1520-0477(1996)077<0437:TNYRP>2.0.CO;2.

Kennedy, A. B., and Coauthors, 2011: Origin of the Hurricane Ike forerunner surge. Geophys. Res. Lett., 38, L08608, doi:10.1029/2011GL047090.

Kirby, J. T., and R. A. Dalrymple, 1983: A parabolic equation for the combined refraction-diffraction of Stokes waves by mildly 
varying topography. J. Fluid Mech., 136, 453-466, doi:10.1017/ S0022112083002232.

—_, and H. T. Ozkan, 1994: REF/DIF S version 1.1: Documentation and user's manual. CACR Rep. 94-04, Center for Applied Coastal Research, University of Delaware, $128 \mathrm{pp}$. [Available online at http://chinacat.coastal.udel.edu/papers/ cacr-94-04.ps.]

Komen, G. J., and K. Hasselmann, 1984: On the existence of a fully developed wind-sea spectrum. J. Phys. Oceanogr., 14, 1271-1285, doi:10.1175/1520-0485(1984)014<1271: OTEOAF $>2.0 . \mathrm{CO} ; 2$.

Landsea, C., and Coauthors, 2004: The Atlantic Hurricane Database Re-Analysis Project: Documentation for the 1851-1910 alterations and additions to the HURDAT database. Hurricanes and Typhoons: Past, Present and Future, R. J. Murnane and K.-B. Liu, Eds., Columbia University Press, 177-221.

_- and Coauthors, 2008: A reanalysis of the 1911-20 Atlantic Hurricane Database. J. Climate, 21, 2138-2168, doi:10.1175/ 2007JCLI1119.1.

Lehner, S., J. Schulz-Stellenfleth, B. Schattler, H. Breit, and J. Horstmann, 2000: Wind and wave measurements using complex ERS-2 SAR wave mode data. IEEE Trans. Geosci. Remote Sens., 38, 2246-2257, doi:10.1109/36.868882.

Mínguez, R., B. G. Reguero, A. Luceño, and F. J. Méndez, 2012: Regression models for outlier identification (hurricanes and typhoons) in wave hindcast databases. J. Atmos. Oceanic Technol., 29, 267-285, doi:10.1175/JTECH-D-11-00059.1.

Palmsten, M. L., and A. Sallenger, 2001: Application of the SWAN wave model to hurricane generated waves on the North Carolina continental shelf. Eos, Trans. Amer. Geophys. Union, 82 (Fall Meet. Suppl.), Abstract OS31B-0416.

Popinet, S., R. M. Gorman, G. J. Rickard, and H. L. Tolman, 2010: A quadtree-adaptive spectral wave model. Ocean Modell., 34, 36-49, doi:10.1016/j.ocemod.2010.04.003.

Powell, M. D., S. H. Houston, N. Amat, and L. R. MorisseauLeroy, 1998: The HRD real-time hurricane wind analysis system. J. Wind Eng. Ind. Aerodyn., 77-78, 53-64, doi:10.1016/ S0167-6105(98)00131-7.

, and Coauthors, 2010: Reconstruction of Hurricane Katrina's wind fields for storm surge and wave hindcasting. Ocean Eng., 37, 26-36, doi:10.1016/j.oceaneng.2009.08.014.
Rattanapitikon, W., and T. Shibayama, 1998: Energy dissipation model for regular and irregular breaking waves. Coastal Eng. J., 40, 327-346, doi:10.1142/S0578563498000194.

Reguero, B., M. Menéndez, F. J. Méndez, R. Mínguez, and I. J. Losada, 2012: A global ocean wave (GOW) calibrated reanalysis from 1948 onwards. Coastal Eng., 65, 38-55, doi:10.1016/ j.coastaleng.2012.03.003.

Ris, R. C., L. H. Holthuijsen, and N. Booij, 1999: A thirdgeneration wave model for coastal regions: 2. Verification. J. Geophys. Res, 104, 7667-7681, doi:10.1029/1998JC900123.

RoSPA, 2005: Safety on European beaches: Operational guidelines. 1st ed. Royal Society for the Prevention of Accidents, $40 \mathrm{pp}$.

Ruiz, A., A. Nistal, B. Pérez, M. Huerta, M. Ruiz, J. Nieto, and O. Serrano, 1995: Base de datos de clima marítimo en España. Ing. Agua, 2, 75-88. [Available online at https://upcommons.upc.edu/ revistes/bitstream/2099/3351/1/25article5.pdf.]

Saha, S., and Coauthors, 2010: The NCEP Climate Forecast System Reanalysis. Bull. Amer. Meteor. Soc., 91, 1015-1057, doi:10.1175/ 2010BAMS3001.1.

Sampson, C. R., P. A. Wittmann, and H. L. Tolman, 2010: Consistent tropical cyclone wind and wave forecasts for the U.S. Navy. Wea. Forecasting, 25, 1293-1306, doi:10.1175/2010WAF2222376.1.

$\longrightarrow,-$, E. A. Serra, H. L. Tolman, J. Schauer, and T. Marchok, 2013: Evaluation of wave forecasts consistent with tropical cyclone warning center wind forecasts. Wea. Forecasting, 28, 287-294, doi:10.1175/WAF-D-12-00060.1.

Silva, R., G. Govaere, P. Salles, G. Bautista, and G. Díaz, 2002: Oceanographic vulnerability to hurricanes on the Mexican coast. Coastal Engineering 2002: Solving Coastal Conundrums; Proceedings of the 28th International Conference, J. McKee Smith, Ed., Vol. 1, World Scientific, 39-51.

Smith, J. M., and D. T. Resio, 1999: STWAVE: Steady-state spectral wave model user's manual for STWAVE. Engineering Research and Development Center Tech. Rep. ERDC/ CHL SR-01-1, USACE, 60 pp.

Thornton, E. B., and R. T. Guza, 1986: Surf zone longshore currents and random waves: Field data and models. J. Phys. Oceanogr., 16, 1165-1178, doi:10.1175/1520-0485(1986)016<1165: SZLCAR $>2.0 . C O ; 2$.

Zijlema, M., 2010: Computation of wind-wave spectra in coastal waters with SWAN on unstructured grids. Coastal Eng., 57, 267-277, doi:10.1016/j.coastaleng.2009.10.011. 